\title{
Epidemiological Study of Various HPV Strains in Cervical Fluid Samples in South-Eastern Iran, 2018-2020
}

\author{
Reza Bahramabadi, MSc'; Zahra Honarvar, MD²; Maryam Iranpour, MD'; Mohammad Kazemi Arababadi, PhD³; Tania Dehesh, \\ PhD $^{4}$; Bahram Dabiri, MD ${ }^{1,5}$; Abbas Mortezaeizadeh Anari, MD'; Sahar Amirpour Rostami, MSc ${ }^{6}$; Mohammad Salajegheh, MSc'; \\ Shahriar Dabiri, MD ${ }^{*}$

\begin{abstract}
'Pathology and Stem Cell Research Center, Afzalipour School of Medicine, Kerman University of Medical Sciences, Kerman, Iran ${ }^{2}$ Department of Obstetrics and Gynecology, Afzalipour School of Medicine, Kerman University of Medical Sciences, Kerman, Iran ${ }^{3}$ Immunology of Infectious Diseases Research Center, Research Institute of Basic Medical Sciences, and Department of Laboratory Sciences, Faculty of Paramedicine, Rafsanjan University of Medical Sciences, Rafsanjan, Iran

${ }^{4}$ Department of Biostatistics and Epidemiology, Faculty of Medicine, Kerman University of Medical Sciences, Kerman, Iran ${ }^{5}$ Department of Pathology, Resident NYU Langone Health, Mineloa, NY, USA

${ }^{6}$ Pharmaceutics Research Centre, Institute of Neuropharmacology, Kerman University of Medical Sciences, Kerman, Iran
\end{abstract}

\begin{abstract}
Background: Uterine cervical malignancy is one of the commonly detected malignancies related to the human papillomavirus (HPV) and is increasing incidentally in developing countries. Therefore, the use of an efficient diagnostic method is required as an effectual step for cervical cancer prevention and treatment. The purpose of the study was to diagnose various types of HPV in the cervical cytology specimens in the South-East of Iran.

Methods: This cross-sectional study was performed on 1079 cervical fluid cytology specimens referred for two years, between 2018-2020. Polymerase chain reaction (PCR) and hybridization (INNO-LiPA HPV Genotyping EXTRA II assay) were used to determine HPV DNA and their genotypes, respectively.

Results: HPV was positive in $37.7 \%$ (407 of 1079) patients with a mean age of $34.62 \pm 8.82$. Among positive cases, $252(62 \%)$ had only one HPV genotype and 155 (38.05\%) had multiplex HPV genotypes, which included 94 (60.7\%), 38 (24.6\%), $18(11.6 \%)$ and $5(3.2 \%)$ cases with two, three, four and five or more genotypes, respectively. The samples with multiple strains revealed 31 HPV genotypes with the four most prevalent being HPV6 (14.7\%), HPV16 (10.9\%), HPV53 (9.6\%) and HPV51 (5.9\%).

Conclusion: HPV infection is the main health challenge for women that requires improved health service programs and appropriate epidemic vaccination.

Keywords: Cervical cancer, HPV genotypes, Human papillomavirus

Cite this article as: Bahramabadi R, Honarvar Z, Iranpour M, Kazemi Arababadi M, Dehesh T, Dabiri B, et al. Epidemiological study of various hpv strains in cervical fluid samples in south-eastern iran, 2018-2020. Arch Iran Med. 2021;24(9):678-683. doi: 10.34172/aim.2021.97
\end{abstract}

Received: November 7, 2020, Accepted: January 13, 2021, ePublished: September 1, 2021

\section{Introduction}

Cervical malignancy ranks the third in prevalence and the fourth in and cause of death among malignancies of women. It has been stated that chronic infection with human papillomavirus (HPV) types is the main cause of cervical dysplasia and then cervical cancer. ${ }^{1,2} \mathrm{HPV}$ is a virus with several limited genes and proteins that can have long-term, significant and irreversible effects on infected human cells and tissues. ${ }^{3}$ Many strains of HPV have been identified, and it has been estimated that 40 of these strains are sexually transmitted. So far, the identified strains associated with a variety of malignancies are 14 types that can cause cervical, oropharyngeal, tonsil, and tongue cancers. ${ }^{4,5}$ A high percentage of cervical cancers are caused by HPV16 and HPV18, accounting for nearly $70 \%$ of cases. ${ }^{6,7} \mathrm{HPV}$ infections are categorized as the most common infections worldwide; however, most cases are transient and do not lead to cervical anomaly: approximately $54 \%$ and $91 \%$ disappear in 1 and 2 years, respectively. ${ }^{8}$

HPV infections are very common: approximately $75 \%-$ $80 \%$ of people who are sexually active become infected with the virus during their lifetime., ${ }^{9,10}$ The HPV infection prevalence varies in females of different ages, but a considerable number of reports have been linked to the women between the ages of 20-30..$^{10,11}$ In many countries, there is a reverse relation between women's age and the rate of HPV spread. Additionally, it has been reported that low-income areas for all age groups were associated with increases in HPV spread. ${ }^{12}$ Previous studies have shown that the prevalence of HPV has a two-point peak: the first is observed in women under 30 years and the second is seen in women aged 55-64 years. ${ }^{13}$

Actually, the distribution of HPV infections is different across countries and various states. ${ }^{14}$ The global report of the spread of HPV in females without cervical disorders 
is $10.4 \%$, which is $8 \%$ in Asia, and in Iran, the variable has been reported from $0.63 \%$ to $36.2 \% .^{15,16}$ Due to the significant difference in reports in Iran from different provinces and the increasing speed of infection of people via different ways (genital, oral, gastrointestinal tract and skin), there is a need for further research and a more detailed examination of samples related to exposed individuals. In order to more accurately identify HPV transmission methods, it is necessary to identify and control infected individuals associated with this dangerous virus. ${ }^{17,18}$ Therefore, this project was designed to determine the prevalence of different HPV strains among Iranian women, who were undergoing routine screening for cervical cancer using INNO-LiPA HPV Genotyping EXTRA II Assay.

\section{Materials and Methods}

In this cross-sectional study, all women who referred to Kerman University clinics in order to have Pap liquid test specimens test and who met the inclusion criteria were included in the study from 2018 to 2020. A total of 1079 cases of Pap liquid test specimens were collected and delivered to the Pathology and Stem Cell Research Center in Afzalipour School of Medicine, during the period 2018-2020. Participants were women selected with the age limit of 15 to 60 years. The exclusion criteria were as follows: previous hysterectomy, vaginal bleeding, symptomatic cervical or vaginal infection, a history of cervical cancer, pregnancy, radiation therapy, and using immunosuppressive drugs. ${ }^{19,20}$ All the women provided written informed consent at the beginning of the research. The cytological criteria included: 1) the presence of koilocytes with raisin type of nuclei, 2) the presence of dysplastic nuclei (LSIL to HSIL), 3) the presence of parakeratosis and 4) inflammatory process.

\section{DNA Extraction and PCR Condition}

HPV-DNA extraction, evaluating and genotyping were performed by experts. Women's specimens were prepared by a gynecologist from the cervix with the Cytobrush, and fixed in liquid base fixative. For DNA derivation, DNA purification and amplification $\mathrm{MN}$ kits (MACHEREY-NAGEL GmbH, Germany) were used according to the manufacturer's directions and the concentration of extract. DNA concentration was assessed in NanoDrop 2000c Spectrophotometer (Isogen Life Science, Veldzigt, the Netherlands). HPV-DNA polymerase chain reaction (PCR) was performed using 5'-CGTCCMAARGGAWACTGATC-3' as forward primer and 5'-GCMCAGGGWCATAAYAATGG-3' as reverse primer. The PCR duplication steps included denaturation stage for 5 minutes at $95^{\circ} \mathrm{C}, 45$ cycles at $90^{\circ} \mathrm{C}$ for 30 seconds, 45 seconds at $56^{\circ} \mathrm{C}$ for, 45 seconds at $72^{\circ} \mathrm{C}$ and an ultimate step at $72^{\circ} \mathrm{C}$ temperature for 5 minutes. In this project, $\beta$-globin were used as the control to validate the PCR condition. Negative (distilled water) and positive controls were used during the PCR test for every 20 samples to exclude possible contamination and to validate the accuracy. For further validation, the PCR products were run on the $2 \%$ agarose gel and visualized by ethidium bromide. Additionally, the positive samples were selected for more analysis. ${ }^{16,21}$

\section{Human Papilloma Virus Screening}

The HPV-DNA genotyping was performed using the INNO-LiPA HPV Genotyping EXTRA II Assay kit from Fujirebio Europe N.V. Company (Belgium). The kit principle was reverse hybridization. In summary, a fragment of the $\mathrm{L} 1$ region called SPF10 of the HPV DNA was protracted by special primers, afterward the biotinylated amplimers were denatured first and then hybridized with particular probes. In addition, to check the quality of the extracted DNA, the human HLADPB1 gene specific primers were added. Subsequently, streptavidin-conjugated alkaline phosphatase was used and then incubated with BCIP/NBT chromogen, to be confirmed by vision. ${ }^{16}$

\section{Statistical Analysis}

Statistical analysis was done using SPSS version 20 software. The relation between categorical variables was evaluated with chi-square and Fisher exact tests. $P<0.05$ was considered to be statistically significant.

\section{Results}

Of 1079 cases for HPV primary testing for any genotypes, 407 cases $(37.7 \%)$ were HPV DNA positive. The total prevalence of HPV was $47 \%(0.45-0.49)$ in the high-risk (HR) group, 34\% (0.32-0.36) in the low-risk group (LR) and $19 \%(0.18-0.20)$ in the potential high risk (PHR) (Figure 1). Figure 2 shows that the most frequent HRHPV genotype pertained to genotype 16 (69 cases, $10.9 \%$ ) and the lowest number to genotypes 18 and 33 (7 cases, 1.1\%). Among the PHR-HPV types, genotype 53 (62 cases, 9.6\%) was the most prevalent while genotype 26

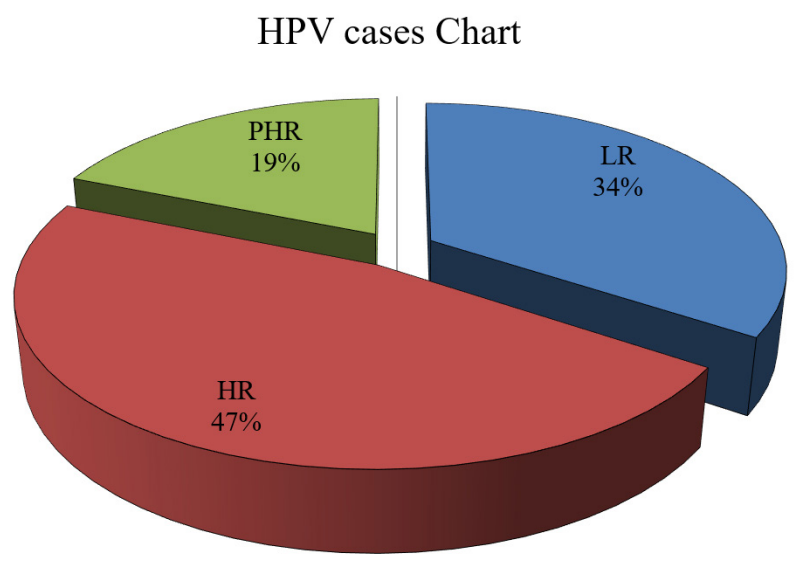

Figure 1. Prevalence of HPV Genotypes. The figure shows that HR (high-risk) HPV was 47\%, the LR (low-risk) HPV 34\%, and PHR (potentially high-risk) HPV of 19\%. The figure also demonstrated that most patients were infected with HR-HPV genotypes and a minority were infected with PHR-HPV genotypes. 


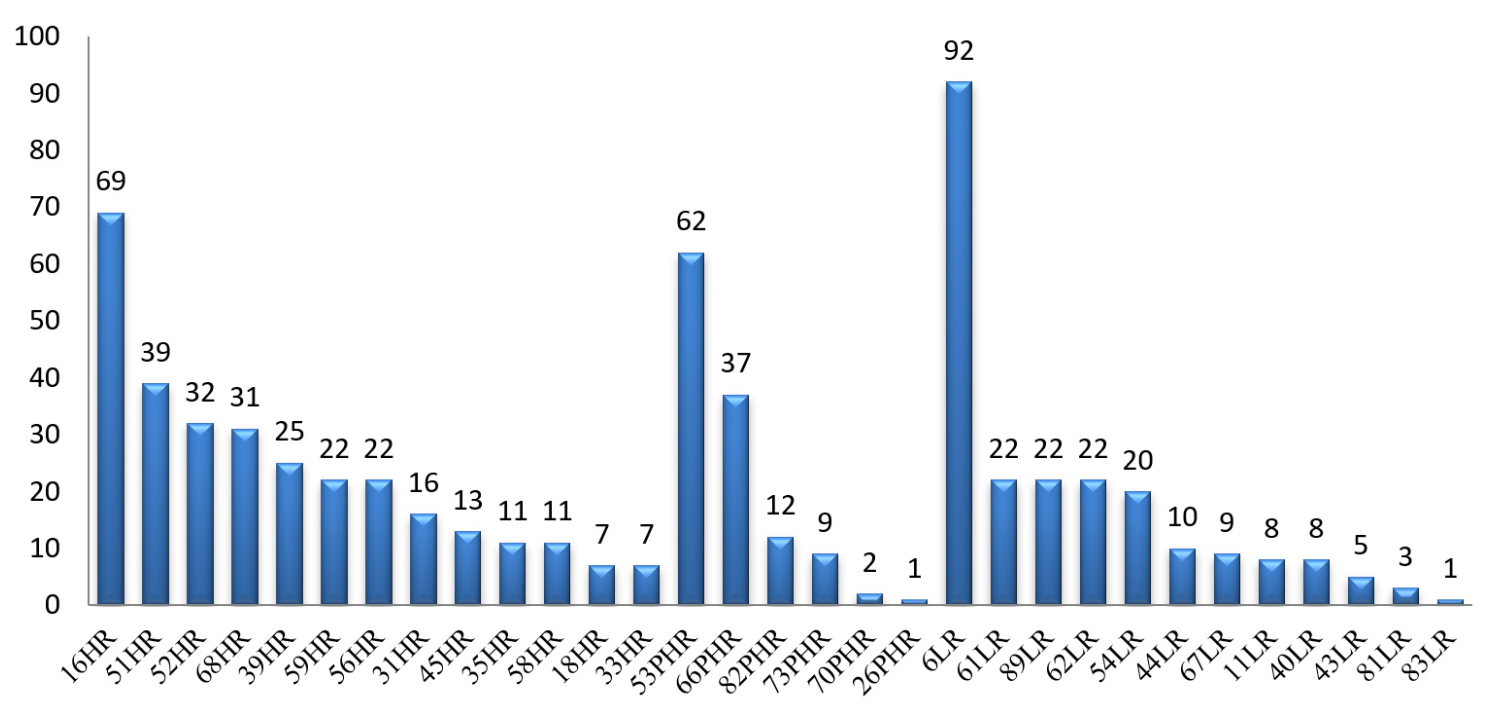

Figure 2. Prevalence of High Risk (HR), Potential High Risk (PHR) and Low Risk (LR) HPV Genotypes. The figure shows that genotype 16 had the highest prevalence, while genotypes 18 and 33 had the lowest prevalence of HR-HPV. Genotype 53 had the highest, while genotype 26 had the lowest prevalence of PHR-HPV and the figure shows that genotype 6 had the highest, while genotype 83 had the lowest prevalence of LR-HPV.

had the lowest prevalence ( 1 case, $0.2 \%)$. Also, genotype 6 (92 cases, $14.7 \%$ ) and 83 ( 1 case, $0.2 \%$ ) were the most and least prevalent LR-HPV subtypes, respectively.

Table 1 shows that there was no significant relation between age and HPV infection status $(P=0.890)$, but the most infected category was 3-40 years (189 cases, 46.4\%). Education status was significantly related to HPV infection status $(P<0.001)$ and the most infected group was high school (209 cases, 51.4\%).

Menopause status was not significantly related to HPV infection $(P=0.859)$. Genotype had a significant relation with HPV infection $(P<0.001)$. Genotype 1 was the most related category among five genotypes. From the cytological point of view in this study, positive predictive correlation between cytological abnormalities (ASCUS, LSIL, HSIL, SCC) and HPV molecular analysis was $87 \%-$ 93\%.

\section{Discussion}

Different types of HPV are responsible for 70\%, 90\%, $60 \%$ and $70 \%$ of vaginal/vulvar, anal, penile and oropharyngeal cancers, respectively. ${ }^{2,22}$ The growth rate of cervical cancer

Table 1. Demographic Characteristics and Four HPV Infection Statuses of the Study Population

\begin{tabular}{|c|c|c|c|c|c|c|}
\hline \multirow{2}{*}{ Variables } & \multirow{2}{*}{ Levels } & \multicolumn{4}{|c|}{ Infection Statuses } & \multirow{2}{*}{$P$ Value } \\
\hline & & HR & PHR & LR & MIX & \\
\hline \multirow{5}{*}{ Age } & $<20$ & 3 & 0 & 1 & 3 & \multirow{5}{*}{0.890} \\
\hline & $21-30$ & 36 & 10 & 27 & 49 & \\
\hline & $31-40$ & 45 & 19 & 51 & 73 & \\
\hline & $41-50$ & 21 & 8 & 20 & 22 & \\
\hline & $>51$ & 4 & 3 & 4 & 7 & \\
\hline \multirow{4}{*}{ Education } & No education & 22 & 0 & 0 & 0 & \multirow{4}{*}{$<0.001$} \\
\hline & Primary education & 59 & 0 & 0 & 0 & \\
\hline & High school & 28 & 40 & 103 & 38 & \\
\hline & University & 0 & 0 & 0 & 117 & \\
\hline \multirow{2}{*}{ Menopause } & Pre menopause & 93 & 32 & 86 & 131 & \multirow{2}{*}{0.859} \\
\hline & Post menopause & 16 & 8 & 17 & 23 & \\
\hline \multirow{5}{*}{ HPV genotypes } & 1 Genotype & 109 & 40 & 103 & 0 & \multirow{5}{*}{$<0.001$} \\
\hline & 2 Genotypes & 0 & 0 & 0 & 94 & \\
\hline & 3 Genotypes & 0 & 0 & 0 & 38 & \\
\hline & 4 Genotypes & 0 & 0 & 0 & 18 & \\
\hline & $\geq 5$ Genotypes & 0 & 0 & 0 & 5 & \\
\hline
\end{tabular}

HR, high risk; PHR, potentially high risk; LR, low risk; HR, high risk; MIX, potentially high risk and low risk 
in developing countries appears to be gradually increasing due to the lack of effective screening programs. ${ }^{16}$ Given that high-risk HPVs appear to be the leading cause of these cancers, rapid diagnosis and treatment of this infection can prevent the progression of the lesions to cancer. The importance of diagnosing this infection was highlighted in routine screening programs and clinical practice. ${ }^{5}$ Global research has shown that HPV-16 and HPV-18 are the most associated single and multi-type infections in cervical cancer. In studies performed on pap smears in Iran, HPV-16 and 18 were identified as the main underlying genotypes. ${ }^{6,8,16}$ Based on a global study on female uterine cervical screening programs that showed an HPV prevalence of $11.7 \%,{ }^{22}$ the prevalence rate of HPV in the present study was (37.7\%) with rates of $47 \%, 19 \%$ and $34 \%$ of subjects respectively placed in the HR, PHR and LR groups. Jamdar et al reported that the prevalence of HR-HPV infection was $10.3 \%{ }^{23}$ in Tehran for cervical cancer routine screening.

Sjoeborg et al mentioned that development of the cancerous area was significantly different in HPV genotypes, with the highest pertaining to genotype HPV16 and then to HPV $-33 .{ }^{24} \mathrm{HPV}$ distribution varies in diverse areas and people, even in different sections of single provinces. According to the Iran National Cancer Registry Center, cervical cancer is the 12 th most common malignancy among Iranian women. ${ }^{15}$ In Iran, a study by Shafaghi et al aimed to determine the genotypes of HPV in cervical samples in the Tehran province: HPV was detected in 265 of 851 patients (31.1\%) and the HPV genotypes detected in the infected samples included HPV$16(7.3 \%)$ and HPV-18 (2.8\%). ${ }^{25}$ In a study by the Isfahan University of Medical Sciences on pap smear of women aged 18-60 years, HPV was diagnosed in 46 samples; 7 HPV-16 (15\%) samples were found. ${ }^{33}$ While HPV-16 is the most common genotype in many countries, HPV-39 in the Northern region of Portugal and HPV-52 and 58 in Shanghai province are predominant genotypes. ${ }^{16,26,27}$ In China, a study by Wang et al to determine the genotypes of HPV revealed that the total HPV positive rate was $14.1 \%{ }^{28}$ The investigation reported that the most prevalent genotypes were HPV-16, HPV-52, HPV58, HPV-53 and HPV-31 with rates of 3.8, 2.5, 1.8, 1.4 and $0.8 \%$, respectively. ${ }^{28}$ The HR-HPV infection has been reported in studies from neighboring Middle-Eastern countries such as Qatar (5.8\%), Saudi Arabia (6.9\%), Kuwait (2.4\%), Pakistan (2.8\%), and Bahrain (9.8\%). ${ }^{29,30}$ However, we found that the positive rate for HPV was $37.7 \%$ and the most prevalent genotypes were HPV-16 (10.9\%), HPV-52 (4.8\%), HPV-53 (9.6\%) and HPV-31 (2.5\%), Single genotype infection was $(38.5 \%)$ and HRHPV infection (47\%). In comparison to our results, a study by Bruni and colleagues demonstrated that the five most prevalent genotypes among a Spanish population were HPV genotypes $16,18,52,31$ and $58,{ }^{15}$ compared to this research, which showed HPV-16 (10.9\%), HPV18 (1.2\%), HPV-52 (4.8\%), HPV-31 (2.5\%), and HPV-
58 (1.8\%). Various studies have been mentioned, ${ }^{15,29,30}$ that are not consistent with our results which may be due to the conditions of the person's sexual partner, the difference in sample size, regional vaccination programs, test methods, etc.

In the present survey, HPV infection peaked at the age of 32 years. There was an elevated occurrence which is probably due to the following: the deficiency of immunity defense versus HPV, absence of an effective vaccination plan at an early age, dangerous unprotected and risky marital sexual behaviors by husband and/or wife, the increasing age of marriage, more coming of women in public swimming pools, increasing cohabitation, having multiple sexual partners, and performing multiple types of waxing and skin abrasions in illegal and unsanitary beauty salons. ${ }^{16,31-33}$ The decrease in the single and multiple HPV infection percentages in middle-aged and older groups, more likely resulting from host immune reaction. ${ }^{1,33}$

The limitations of this study should be noted. The study could not be representativeness of the target population because only the southeastern region was included. Moreover, adjustment of confounders was not done because of the lack of information (like the number of sexual partners, water-pipe smoking, cigarette smoking, etc). Therefore, larger and prospective studies are required to validate our results.

In conclusion, there is a high spread of HPV in Iranian females and like other parts of the world, it might be one of the most important reasons for various cellular changes in the uterine cervical lesion screening programs (ASCUS, AGUS, CIN1, CIN2, CIN3, carcinoma). Therefore, the use of new and different diagnostic methods has the ability to prevent and control early changes in proliferative disorders. Also, by identifying common HPV genotypes in each region of Iran, a suitable vaccine could be developed to control and prevent this type of cellular change.

\section{Authors' Contribution}

$\mathrm{RB}$ and SHD conceived the original idea. MI and ZH selected and investigated the enrolled cases. AMA, SAR and MS carried out the experiment under the supervision of SHD. TD carried out the statistical analysis. BD and MKA wrote the manuscript with support from SHD.

\section{Conflict of Interest Disclosures}

The authors affirmed, No Conflict of Interest.

\section{Ethical Statement}

The Ethical panel of the Kerman University of Medical Sciences has confirmed approved the protocol of this research by under the code IR.KMU.REC.1398.552.

\section{Acknowledgements}

The authors wish to thank the staff of Pathology and Stem Cell Research Center and Donya Dabiri DDM for their assistance technical support in this study. This article was extracted from the RB student thesis. 


\section{References}

1. Doorbar J. Host control of human papillomavirus infection and disease. Best Pract Res Clin Obstet Gynaecol. 2018;47:27-41. doi: 10.1016/j.bpobgyn.2017.08.001.

2. Torre LA, Islami F, Siegel RL, Ward EM, Jemal A. Global cancer in women: burden and trends. Cancer Epidemiol Biomarkers Prev. 2017;26(4):444-57. doi: 10.1158/10559965.epi-16-0858.

3. Longatto-Filho A, Syrjänen K. HPV: a virus-dinosaur living among us? Acta Cytol. 2019;63(2):83-4. doi: 10.1159/000497814.

4. Bihl MP, Tornillo L, Kind AB, Obermann E, Noppen C, Chaffard R, et al. Human papillomavirus (HPV) detection in cytologic specimens: similarities and differences of available methodology. Appl Immunohistochem Mol Morphol. 2017;25(3):184-9. doi: 10.1097/pai.0000000000000290.

5. Chan CK, Aimagambetova G, Ukybassova T, Kongrtay K, Azizan A. Human papillomavirus infection and cervical cancer: epidemiology, screening, and vaccination-review of current perspectives. J Oncol. 2019;2019:3257939. doi: 10.1155/2019/3257939.

6. Bahramabadi R, Dabiri S, Iranpour M, Kazemi Arababadi M. TLR4: an important molecule participating in either antihuman papillomavirus immune responses or development of its related cancers. Viral Immunol. 2019;32(10):417-23. doi: 10.1089/vim.2019.0061.

7. WolffE, Elfström KM, Haugen Cange H, Larsson S, Englund H, Sparén P, et al. Cost-effectiveness of sex-neutral HPVvaccination in Sweden, accounting for herd-immunity and sexual behaviour. Vaccine. 2018;36(34):5160-5. doi: 10.1016/j.vaccine.2018.07.018.

8. Burd EM. Human papillomavirus and cervical cancer. Clin Microbiol Rev. 2003;16(1):1-17. doi: 10.1128/cmr.16.1.117.2003.

9. Koutsky LA, Galloway DA, Holmes KK. Epidemiology of genital human papillomavirus infection. Epidemiol Rev. 1988;10:122-63. doi: 10.1093/oxfordjournals.epirev. a 036020 .

10. Serrano B, Brotons M, Bosch FX, Bruni L. Epidemiology and burden of HPV-related disease. Best Pract Res Clin Obstet Gynaecol. 2018;47:14-26. doi: 10.1016/j. bpobgyn.2017.08.006.

11. Weaver B, Shew M, Qadadri B, Tu W, Tong Y, Denski C, et al. Low-level persistence of human papillomavirus 16 DNA in a cohort of closely followed adolescent women. J Med Virol. 2011;83(8):1362-9. doi: 10.1002/jmv.22116.

12. Franceschi S, Herrero R, Clifford GM, Snijders PJ, Arslan A, Anh PT, et al. Variations in the age-specific curves of human papillomavirus prevalence in women worldwide. Int J Cancer. 2006;119(11):2677-84. doi: 10.1002/ijc.22241.

13. Muñoz N, Méndez F, Posso H, Molano M, van den Brule AJ, Ronderos $\mathrm{M}$, et al. Incidence, duration, and determinants of cervical human papillomavirus infection in a cohort of Colombian women with normal cytological results. J Infect Dis. 2004;190(12):2077-87. doi: 10.1086/425907.

14. Zhao P, Liu S, Zhong Z, Hou J, Lin L, Weng R, et al. Prevalence and genotype distribution of human papillomavirus infection among women in northeastern Guangdong province of China. BMC Infect Dis. 2018;18(1):204. doi: 10.1186/s12879-018-3105-x.

15. Bruni L, Diaz M, Castellsagué X, Ferrer E, Bosch FX, de Sanjosé S. Cervical human papillomavirus prevalence in 5 continents: meta-analysis of 1 million women with normal cytological findings. J Infect Dis. 2010;202(12):1789-99. doi: 10.1086/657321.

16. Mobini Kesheh M, Keyvani H. The prevalence of HPV genotypes in Iranian population: an update. Iran J Pathol. 2019;14(3):197-205. doi: 10.30699/ijp.2019.90356.1861.

17. de Sanjosé S, Diaz M, Castellsagué X, Clifford G, Bruni L, Muñoz N, et al. Worldwide prevalence and genotype distribution of cervical human papillomavirus DNA in women with normal cytology: a meta-analysis. Lancet Infect Dis. 2007;7(7):453-9. doi: 10.1016/s1473-3099(07)70158-5.

18. Malary M, Moosazadeh M, Hamzehgardeshi Z, Afshari M, Moghaddasifar I, Afsharimoghaddam A. The prevalence of cervical human papillomavirus infection and the most atrisk genotypes among Iranian healthy women: a systematic review and meta-analysis. Int J Prev Med. 2016;7:70. doi: 10.4103/2008-7802.181756.

19. De Vuyst H, Claeys P, Njiru S, Muchiri L, Steyaert S, De Sutter $\mathrm{P}$, et al. Comparison of pap smear, visual inspection with acetic acid, human papillomavirus DNA-PCR testing and cervicography. Int J Gynaecol Obstet. 2005;89(2):1206. doi: 10.1016/j.ijgo.2005.01.035.

20. Khodakarami N, Farzaneh F, Aslani F, Alizadeh K. Comparison of Pap smear, visual inspection with acetic acid, and digital cervicography as cervical screening strategies. Arch Gynecol Obstet. 2011;284(5):1247-52. doi: 10.1007/s00404-010-1793-6.

21. Falaki F, Amir Chaghmaghi M, Pakfetrat A, Delavarian Z, Mozaffari PM, Pazooki N. Detection of human papilloma virus DNA in seven cases of focal epithelial hyperplasia in Iran. J Oral Pathol Med. 2009;38(10):773-6. doi: 10.1111/j.1600-0714.2009.00784.x.

22. Torre LA, Bray F, Siegel RL, Ferlay J, Lortet-Tieulent J, Jemal A. Global cancer statistics, 2012. CA Cancer J Clin. 2015;65(2):87-108. doi: 10.3322/caac.21262.

23. Jamdar F, Farzaneh F, Navidpour F, Younesi S, Balvayeh $\mathrm{P}$, Hosseini $\mathrm{M}$, et al. Prevalence of human papillomavirus infection among Iranian women using COBAS HPV DNA testing. Infect Agent Cancer. 2018;13:6. doi: 10.1186/ s13027-018-0178-5.

24. Sjoeborg KD, Tropé A, Lie AK, Jonassen CM, Steinbakk M, Hansen M, et al. HPV genotype distribution according to severity of cervical neoplasia. Gynecol Oncol. 2010;118(1):29-34. doi: 10.1016/j.ygyno.2010.03.007.

25. Shafaghi B, Jarollahi A, Yousefzadeh B, Ameri A, Moghadam S, Mostafavi M. Human papilloma virus prevalence and types among Iranian women attending regular gynecological visits. Rep Radiother Oncol. 2013;1(2):e2389.

26. Huang S, Afonina I, Miller BA, Beckmann AM. Human papillomavirus types 52 and 58 are prevalent in cervical cancers from Chinese women. Int J Cancer. 1997;70(4):408- 
11. doi: 10.1002/(sici)1097-0215(19970207)70:4<408::aidijc6>3.0.co;2-\#.

27. Sousa H, Tavares A, Campos C, Marinho-Dias J, Brito M, Medeiros R, et al. High-risk human papillomavirus genotype distribution in the Northern region of Portugal: data from regional cervical cancer screening program. Papillomavirus Res. 2019;8:100179. doi: 10.1016/j. pvr.2019.100179.

28. Wang J, Tang D, Wang K, Wang J, Zhang Z, Chen Y, et al. HPV genotype prevalence and distribution during 20092018 in Xinjiang, China: baseline surveys prior to mass HPV vaccination. BMC Womens Health. 2019;19(1):90. doi: 10.1186/s12905-019-0785-3.

29. Bansal D, Elmi AA, Skariah S, Haddad P, Abu-Raddad LJ, Al Hamadi AH, et al. Molecular epidemiology and genotype distribution of human papillomavirus (HPV) among Arab women in the State of Qatar. J Transl Med. 2014;12:300. doi: 10.1186/s12967-014-0300-4.

30. Sohrabi A, Hajia M. Cervical cancer and genital infections: assessment of performance and validation in human papillomavirus genotyping assays in Iran, its neighbouring countries and Persian Gulf area. Iran J Pathol. 2017;12(1):35-44.

31. Kirchhof MG, Au S. Brazilian waxing and human papillomavirus: a case of acquired epidermodysplasia verruciformis. CMAJ. 2015;187(2):126-8. doi: 10.1503/ cmaj.140198.

32. Nyitray AG, da Silva RJ, Baggio ML, Lu B, Smith D, Abrahamsen M, et al. The prevalence of genital HPV and factors associated with oncogenic HPV among men having sex with men and men having sex with women and men: the HIM study. Sex Transm Dis. 2011;38(10):932-40. doi: 10.1097/OLQ.0b013e31822154f9.

33. Allameh T, Moghim S, Farahbod F. Reviewing the prevalence of human papillomavirus (HPV) in married women aged 18-60 years with normal Pap smear referring to gynecology clinics in hospitals affiliated to Isfahan University of Medical Sciences, Iran. J Isfahan Med Sch. 2012;29(163):1-8. 